\title{
On rough convergence variables of triple sequences
}

\author{
M. Kemal Ozdemir ${ }^{1, *}$, Ayhan $\mathrm{Esi}^{2, * *}$, and Ayten $\mathrm{Esi}^{2, * * *}$ \\ ${ }^{1}$ Department of Mathematics, İnönü University, 44280, Malatya, Turkey \\ ${ }^{2}$ Department of Mathematics, Adiyaman University, 02040, Adıyaman, Turkey
}

\begin{abstract}
Triple sequence convergence has an extremly important position in the basic theory of mathematics. The present manuscript contains four types of convergence concept of convergence almost surely, convergence incredibility, trust convergence in mean and convergence in distribution and discuss the relation ship among those and some mathematical properties of those new convergence.
\end{abstract}

\section{Introduction}

Initially Phu [8-10] has presented the concept for the rough convergence in a finite dimensional normed spaces. He also illustrated the fact that this set $\operatorname{LIM}_{x}^{r}$ is closed, bounded and convex; at the same time presented the concept for the rough Cauchy sequence. He again studied the connections among rough convergence and the other types of convergence and also the dependence for $L I M_{x}^{r}$ on the roughness having degree of $r$.

Aytar [1] investigated rough statistical convergence and gave the definition for the set of rough statistical limit points of a sequence and also got two important statistical convergence criteria related to this set and show that it is convex and closed set. Furthermore, Aytar [2] examined that this $r$-limit set of the sequence equals to intersection of those sets and also that $r$-core of the sequence equals to the union of those sets. Dundar and Cakan [5] studied about rough ideal convergence and defined the set of rough ideal limit points for a given sequence.

In the present manuscript, we present the concept of rough convergence and the set of rough limit points of a triple sequence and get two rough convergence criteria related to this set, also show that the set is closed and convex and investigated the relations among the set of accumulation points and the set of rough limit points of a triple sequence.

A triple sequence (either complex or real) can also be described via a function defined as $x: \mathbb{N}^{3} \rightarrow \mathbb{R}(\mathbb{C})$, in which the natural, real and complex numbers are denoted by $\mathbb{N}, \mathbb{R}$ and $\mathbb{C}$, respectively. The various types of concepts for triple sequence has been presented and examined initially by Datta et al. [3], Debnath et al. [4], Esi et al. [6, 7], Sahiner et al. [11] and several others.

Consider $\left(x_{m n k}\right)$ is a triple sequence of rough variables. In this paper we discuss some convergence notions of rough triple sequence: convergence almost surely (a.s.), convergence in credibility, trust convergence in mean and also in distribution and some mathematical properties among them.

\footnotetext{
*e-mail: kozdemir73@gmail.com

**e-mail: aesi23@hotmail.com

***e-mail: aytenesi@yahoo.com
} 


\section{Definitions and Preliminaries}

Definition 2.1 It is said that a triple sequence $x=\left(x_{m n k}\right)$ is rough convergent ( $r$-convergent) to $l$, denoted as $x_{m n k} \rightarrow^{r}$ l, under the assumption that

$$
\forall \epsilon>0, \exists i_{\epsilon} \in \mathbb{N}: m, n, k \geq i_{\epsilon} \Longrightarrow\left|x_{m n k}-l\right|<r+\epsilon,
$$

or equivalently, if

$$
\lim \sup \left|x_{m n k}-l\right| \leq r .
$$

where $r$ is known as the roughness of degree. When $r=0$ is taken, the ordinary convergence of a triple sequence is obtained.

Definition 2.2 Let a triple sequence of rough variables $\left(x_{m n k}\right)$ is said to be convergent almost surely to the rough variable $l$ iff there is a set $A \in \mathbb{A}$ with $\operatorname{Tr}(A)=1$ such that $\lim _{u v w \rightarrow \infty}\left|x_{m n k}(\lambda)-l(\lambda)\right|=0$ for every $\lambda \in A$. Under these conditions, we can write $x_{m n k} \rightarrow^{\text {a.s. }} l$.

Definition 2.3 Let us assume that $r$ is a real number which is non-negative and $\left(x_{m n k}\right)$ be a triple sequence of rough variables. We say that the triple sequence $\left(x_{m n k}\right)$ converges in trust to the rough variable l if $\lim _{m n k \rightarrow \infty} \operatorname{Tr}\left\{\left|x_{m n k}-l\right| \geq r+\epsilon\right\}=0$ for every $\epsilon>0$. Under these conditions, we can also write $\left(x_{m n k}\right) \rightarrow_{r}^{T r v} l$.

Definition 2.4 Let $r$ be any real number which is non-negative and $\left(x_{m n k}\right)$ be a triple sequence of rough variables having finite expected values, the triple sequence $\left(x_{m n k}\right)$ converges in mean to the rough variable lif $\lim _{m n k \rightarrow \infty} E\left[\left|x_{m n k}-l\right|\right]=0$. Under these conditions, we can write $\left(x_{m n k}\right) \rightarrow^{E} l$.

Definition 2.5 Let us assume that $\Phi, \Phi_{1}, \Phi_{2}, \ldots$, are the trust distributions of rough variables $l, l_{1}, l_{2}, \ldots$, respectively. If the triple sequence $\left(\Phi_{m n k}\right)$ converges weakly to $\Phi$, then we say that $\left(x_{m n k}\right) \rightarrow^{\text {Dis }} l$.

\section{Main Results}

Theorem 3.1 Let us $r$ be a real number which is non-negative and $\left(x_{m n k}\right)$ be a triple sequence of rough variables, then $\left(x_{m n k}\right) \rightarrow^{\text {a.s. }} l \Longleftrightarrow$ for every $\epsilon>0$, we have

$$
\lim _{u v w \rightarrow \infty} \operatorname{Tr}\left\{\bigcup_{(m, n, k)=(u, v, w)}\left\{\left|x_{m n k}-l\right| \geq r+\epsilon\right\}\right\}=0
$$

Proof. For every $(m, n, k) \geq 1$ and $\epsilon>0$, we describe

$$
X=\left\{\lambda \in \Lambda: \lim _{m n k \rightarrow \infty} x_{m n k}(\lambda) \neq l(\lambda)\right\}, X=\left\{\lambda \in \Lambda:\left|x_{m n k}(\lambda)-l(\lambda)\right| \geq r+\epsilon\right\} .
$$

It is clear that

$$
X=\bigcup_{\epsilon>0}\left(\bigcap_{(u, v, w)=1}^{\infty} \bigcup_{(m, n, k)=(u, v, w)}^{\infty} X_{m n k}(r+\epsilon)\right) .
$$

Note that $x_{m n k} \rightarrow{ }_{r}^{a . s .} l$, if and only if $\operatorname{Tr}(X)=0$, i.e.,

$$
x_{m n k} \rightarrow_{r}^{a . s .} l \Longleftrightarrow \operatorname{Tr}\left\{\bigcap_{(u, v, w)=1}^{\infty} \bigcup_{(m, n, k)=(u, v, w)}^{\infty} X_{m n k}(r+\epsilon)\right\}=0
$$


for every $\epsilon>0$. Since,

$$
\bigcup_{(m, n, k)=(u, v, w)}^{\infty} X_{m n k}(r+\epsilon) \downarrow \bigcap_{(u, v, w)=1}^{\infty} \bigcup_{(m, n, k)=(u, v, w)}^{\infty} X_{m n k}(r+\epsilon)
$$

it is followed from the continuity of trust measure that

$$
\lim _{u v w \rightarrow \infty} \operatorname{Tr}\left\{\bigcup_{(m, n, k)=(u, v, w)}^{\infty} X_{m n k}(r+\epsilon)\right\}=\operatorname{Tr}\left\{\bigcap_{(u, v, w)=1}^{\infty} \bigcup_{(m, n, k)=(u, v, w)}^{\infty} X_{m n k}(r+\epsilon)\right\} .
$$

Theorem 3.2 Let us assume that $r$ is a real number which is non-negative and $\left(x_{m n k}\right)$ be a triple sequence of rough variables. If $\left(x_{m n k}\right) \rightarrow_{r}^{a . s .} l$, then $\left(x_{m n k}\right) \rightarrow_{r}^{T r} l$.

Proof. It is followed from both the convergence a.s. and also Theorem 3.1 that

$$
\lim _{u v w \rightarrow \infty} \operatorname{Tr}\left\{\bigcup_{(m, n, k)=(u, v, w)}^{\infty}\left\{\left|x_{m n k}-l\right| \geq r+\epsilon\right\}\right\}=0
$$

for each $\epsilon>0$. For every $(u, v, w) \geq 1$, since we have

$$
\left\{\left|x_{u v w}-l\right| \geq r+\epsilon\right\} \subset \bigcup_{(m, n, k)=(u, v, w)}^{\infty}\left\{\left\{\left|x_{m n k}-l\right| \geq r+\epsilon\right\}\right\}
$$

the theorem holds.

Theorem 3.3 Let us assume that $r$ be a real number which is non-negative and $\left(x_{m n k}\right)$ be a triple sequence of rough variables. If the triple sequence $\left(x_{m n k}\right) \rightarrow_{r}^{\text {mean }} l$, then $\left(x_{m n k}\right) \rightarrow_{r}^{T r} l$.

Proof. For any given number $\epsilon>0$

$$
\operatorname{Tr}\left\{\left|x_{m n k}-l\right| \geq r+\epsilon\right\} \leq \frac{E\left[\left|x_{m n k}-l\right|\right]}{r+\epsilon} \rightarrow 0 \text { as } m, n, k \rightarrow \infty .
$$

Thus $\left(x_{m n k}\right) \rightarrow_{r}^{T r} l$.

Thus trust distribution $\Phi: \mathbb{R}^{3} \rightarrow[0,1]$ of a rough variable $l$ is described as

$$
\Phi(x)=\operatorname{Tr}\{\lambda \in \Lambda: l(\lambda) \leq x\} \text { for all } x \in \mathbb{R}^{3}
$$

i.e., $\Phi(x)$ is the trust having the rough variable $l \leq x$.

Theorem 3.4 Suppose that rough variables are $l, l_{1}, l_{2}, \ldots$. When triple sequence $\left(x_{m n k}\right) \rightarrow_{r}^{T r}$ $l$, then $\left(x_{m n k}\right) \rightarrow{ }_{r}^{\text {Dist }} l$.

Proof. Let us assume $x$ is a prescribed continuity point in the distribution $\Phi$. Again, On the other side, when $y>x$, we have

$$
\begin{gathered}
\left\{x_{m n k} \leq x\right\}=\left\{x_{m n k} \leq x, l<y\right\} \bigcup\left\{x_{m n k} \leq x, l>y\right\} \subset\{l<y\} \bigcup\left\{\left|x_{m n k}-l\right| \geq y-x\right\} \\
\Longrightarrow \Phi_{m n k}(x) \leq \Phi(y)+\operatorname{Tr}\left\{\left|x_{m n k}-l\right| \geq y-x\right\} .
\end{gathered}
$$


Since $\left(x_{m n k}\right) \rightarrow{ }_{r}^{T r} l$, we have

$$
\operatorname{Tr}\left\{\left|x_{m n k}-l\right| \geq y-x\right\} \rightarrow 0 .
$$

Therefore we have

$$
\lim _{m, n, k \rightarrow \infty} \sup \Phi_{m n k}(x) \leq \Phi(y) \text { for every } y>x .
$$

If we let $y \rightarrow x$, then we have

$$
\lim _{m, n, k \rightarrow \infty} \sup \Phi_{m n k}(x) \leq \Phi(x) .
$$

Similarly, on the way around, when $z<x$ is taken, we obtain

$$
\begin{gathered}
\{l \leq z\}=\left\{l \leq z, x_{m n k} \leq x\right\} \bigcup\left\{l \leq z, x_{m n k}>x\right\} \\
\subset\left\{x_{m n k} \leq x\right\} \bigcup\left\{\left|x_{m n k}-l\right| \geq x-z\right\} \\
\Longrightarrow \Phi(z) \leq \Phi_{m n k}(x)+\operatorname{Tr}\left\{\left|x_{m n k}-l\right| \geq x-z\right\} .
\end{gathered}
$$

Since $\operatorname{Tr}\left\{\left|x_{m n k}-l\right| \geq x-z\right\} \rightarrow 0$, we get $\Phi(z) \leq \lim _{m n k \rightarrow \infty} \inf \Phi_{m n k}(x)$ for the condition $z<x$. If we let $z \rightarrow x$, then we have

$$
\Phi(x) \leq \lim _{m n k \rightarrow \infty} \inf \Phi_{m n k}(x) .
$$

This is followed directly from Eqs. (3.1)-(3.2) we get $\Phi_{m n k}(x) \rightarrow \Phi(x)$.

Conclusion: This paper contributed to the research area of rough variable triple sequence were defined and then some mathematical properties of those new convergence are discussed.

\section{References}

[1] Aytar S., Rough statistical convergence, Numer. Funct. Anal. Optim. 29(3-4), 291-303 (2008).

[2] Aytar S., The rough limit set and the core of a real sequence, Numer. Funct. Anal. Optim. 29(3-4), 283-290 (2008).

[3] Datta, A. J.; Esi, A. and Tripathy, B. C., Statistically convergent triple sequence spaces defined by Orlicz function, J. Math. Anal. 4(2), 16-22 (2013).

[4] Debnath S., Sarma, B. and Das, B. C., Some generalized triple sequence spaces of real numbers, J. Nonlinear Anal. Optim. 6(1), 71-79 (2015).

[5] Dundar, E.; Cakan, C., Rough I-convergence, Demonstr. Math.47(3), 638-651 (2014).

[6] Esi, A., On some triple almost lacunary sequence spaces defined by Orlicz functions, Research and Reviews: Discrete Mathematical Structures 1(2), 16-25 (2014).

[7] Esi, A. and Catalbas M. N., Almost convergence of triple sequences, Global Journal of Mathematical Analysis 2(1), 6-10 (2014).

[8] Phu, H. X., Rough convergence in normed linear spaces, Numer. Funct. Anal. Optim. 22(1-2), 199-222 (2001).

[9] Phu H. X., Rough continuity of linear operators, Numer. Funct. Anal. Optim. 23(1-2), 139-146 (2002).

[10] Phu H. X., Rough convergence in infinite dimensional normed spaces, Numer. Funct. Anal. Optimiz. 24(3-4), 285-301 (2003).

[11] Sahiner, A.; Gurdal, M. and Duden F. K., Triple sequences and their statistical convergence, Selcuk J. Appl. Math. 8(2), 49-55 (2007). 